\title{
THE SMITHSONIAN ASTROPHYSICAL OBSERVATORY
}

$\mathrm{M}$ ORE than 350 astronomers representing approximately twenty countries will participate in two space-science conferences to be held at Cambridge, Massachusetts, this summer as a part of a celebration marking the seventy-fifth anniversary of the Smithsonian Astrophysical Observatory.

The Smithsonian Observatory, in co-operation with the Harvard College Observatory, will sponsor two symposia, the first concerning meteor orbits and dust (August 9-13), and the second concerning aeronomy, or the investigation of the Earth's upper atmosphere by means of artificial satellites (August 16-20). The Smithsonian Observatory has long been a centre for research in both aeronomy and meteoritics, and specialists in these fields from around the world are expected to attend, including scientists from Iron Curtain countries.

Two popular lectures open to the public will also be delivered: the first on August 10, entitled "The Falling Stars", given by Dr. Peter Millman of the National Research Council of Canada, and the second on August 17, entitled "The Aurora Polaris in the Magnetosphere", given by Dr. Sydney Chapman of the Geophysical Institute, University of Alroska.

The Smithsonian Astrophysical Observatory, a bureau of the Smithsonian Institution, Washington, D.C., was founded in 1890 by Samuel Pierpont Langley, third secretary of the Smithsonian and a pioneer in aeronautics and early manned flight. The Observatory's early research was concerned with the relationship between solar and geophysical phenomena.

In 1955, the Observatory headquarters and research facilities were moved to the grounds of the Harvard College Observatory in Cambridge, Massachusetts. Dr. Fred L. Whipple, the well-known Harvard astronomer, was appointed director of the Observatory at that time. The emphasis of Smithsonian research was shifted to the 'space sciences' in response to growing international space programmes.

The Observatory gained widespread recognition with the launching of Russia's Sputnik I. The Observatory was developing a world-wide network of satellite-tracking cameras at the time, and it already had an international organization of volunteer visual observers in operation. This group of amateur observers, called "Moonwatch", provided many of the first data leading to the determination of Sputnik's orbit.

The Smithsonian Observatory has continued its tracking operations under a grant from the National Aeronautics and Space Administration. Optical data from its network of Baker-Nunn cameras are used to-day both in support of the federal space programme and for the research of its own scientists who use the information in investigations of the upper atmosphere, celestial mechanies, the Earth's gravitational potential, and geodesy.

Besides satellite tracking and its related research projects, more than fifty Observatory scientists are engaged in varied researches ranging from theoretical astrophysics and meteoritics to exobiology and cometary studies. As part of the National Aeronautics and Space Administration's Orbiting Observatory programme, Smithsonian scientists are also preparing instrumentation for future satellite and balloon flights.

The Smithsonian Observatory's data-gathering facilities include the world-wide network of twelve Baker-Nunn satellite-tracking cameras, an unmanned network of sixteen camera stations in the mid-western United States for photographing bright meteors, a system of radar and optical tracking stations on the Virginia coast for observing simulated or 'man-made meteors', and, with the Harvard College Observatory, a radar system at Havana, Illinois, for the detection of ionized trails from micrometeorites.

\section{LACK OF HOMOLOGY IN THE OSCILLATIONS OF NEUTRON STARS}

\author{
By DR. SACHIKO TSURUTA
}

\author{
Smithsonian Institution Astrophysical Observatory, Cambridge, Massachusetts
}

$\mathrm{T}$

HE examination of highly condensed matter is important in itself as a fundamental problem in physics. Its importance has recently been increased because of a possibility that the presence of dense stellar matter in the form of a neutron star may be responsible for some of the celestial X-ray sources now known to exist. Oppenheimer and Volkoff ${ }^{1}$ discussed the dynamical stability of neutron stars by calculating free energies. A variational principle was used by Misner and Zapolsky ${ }^{2}$ for such investigations. Both approaches were used by Wheeler et $a l .{ }^{3-5}$, who extended the problem to more general cases. This article presents some results of recent calculations which show how various possible forms of the nuclear potential are related to the general stability of highdensity objects.

For stars to be stable they must first of all be in hydrostatic equilibrium. Models were, therefore, constructed by solving the general relativistic equations of hydrostatic equilibrium as derived by Oppenheimer and Volkoff ${ }^{1}$. In order to estimate the dynamical stability of these models, Chandrasekhar's variational principle ${ }^{6-8}$ (in its corrected form) was used. According to this principle, a sufficient condition for the occurrence of dynamical instability is that the square of the circular frequency of a radial oscillation, $\omega^{2}$, be negative. Chandrasekhar's trial function, which assumes homologous oscillations, was used.

Both the expressions for the hydrostatic equilibrium and for the dynamical stability contain the equation of state, the relation between the pressure and energy density, $P=P(\varepsilon)$. It may be noted that the effect of nuclear forces enters our problem through this relation. When the stellar density approaches the normal nuclear density $\left(3.7 \times 10^{14} \mathrm{~g} / \mathrm{cm}^{3}\right)$, the constituent particles generally experience attractive forces; but as the density increases further, repulsive forces eventually predominate. To see the effect of these forces, three different types of nuclear potential were chosen. The first, originally developed by Skyrme ${ }^{9}$, is a simple three-body effective 